See discussions, stats, and author profiles for this publication at: https://www.researchgate.net/publication/337429969

\title{
Examining the Discriminant Validity of Complex Posttraumatic Stress Disorder and Borderline Personality Disorder Symptoms: Results From a United Kingdom Population Sample
}

Article in Journal of Traumatic Stress · November 2019

DOI: $10.1002 /$ its.22444

CITATIONS

13

4 authors:

Philip Hyland

92 National University of Ireland, Maynooth 216 PUBLICATIONS 2,936 CITATIONS

SEE PROFILE

Mark Shevlin

Ulster University

416 PUBLICATIONS 9,902 CITATIONS

SEE PROFILE

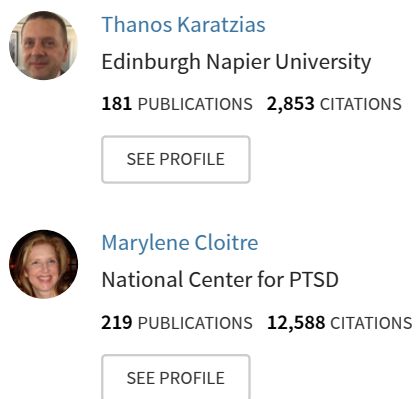

Some of the authors of this publication are also working on these related projects: 


\title{
Examining the Discriminant Validity of Complex Posttraumatic Stress Disorder and Borderline Personality Disorder Symptoms: Results From a United Kingdom Population Sample
}

\author{
Philip Hyland (D), 1,2 Thanos Karatzias, ${ }^{3,4}$ Mark Shevlin (D) 5 and Marylène Cloitre ${ }^{6,7}$ \\ ${ }^{1}$ Department of Psychology, Maynooth University, Kildare, Ireland \\ ${ }^{2}$ Centre for Global Health, Trinity College Dublin, Dublin, Ireland \\ ${ }^{3}$ National Health Service Lothian, Rivers Centre for Traumatic Stress, Edinburgh, United Kingdom \\ ${ }^{4}$ School of Health and Social Care, Edinburgh Napier University, Edinburgh, United Kingdom \\ ${ }^{5}$ School of Psychology, Ulster University, Derry, Northern Ireland \\ ${ }^{6}$ National Center for PTSD Dissemination and Training Division, VA Palo Alto Health Care System, Palo Alto, California, USA \\ ${ }^{7}$ Department of Psychiatry and Behavioral Sciences, Stanford University, Stanford, California, USA
}

\begin{abstract}
Complex posttraumatic stress disorder (CPTSD) was added to the diagnostic nomenclature in the 11th revision of the International Classification of Diseases (ICD-11). Although considerable evidence exists supporting the construct validity of CPTSD, the distinguishability of CPTSD symptoms from those of borderline personality disorder (BPD) has been questioned. The present study examined the discriminant validity of CPTSD and BPD symptoms among a trauma-exposed population sample from the United Kingdom $(N=546)$. Participants completed self-report measures of CPTSD and BPD symptoms, and their latent structure was assessed using exploratory structural equation modeling (ESEM). A three-factor model with latent variables reflecting PTSD, disturbances in self-organization (DSO), and BPD symptoms provided the best fit of the data, $\chi^{2}(399, N=546)=1,650, p<.001 ; \mathrm{CFI}=.944 ;$ TLI $=.930 ; \mathrm{RMSEA}=.077,90 \% \mathrm{CI}$ $[.073, .081]$. We identified multiple symptoms distinctive to individual constructs (e.g., disturbed relationships and suicidality) as well as symptoms shared across the constructs (e.g., affective dysregulation). The PTSD, $\beta=.24$; DSO, $\beta=.23$; and BPD, $\beta=.27$, latent variables were positively and significantly associated with childhood interpersonal trauma. The current findings support the discriminant validity of CPTSD and BPD symptoms and highlight various phenomenological signatures of each construct as well as demonstrate how these constructs share important similarities in symptom composition and exogenous correlates.
\end{abstract}

In contrast to the expanded definition of posttraumatic stress disorder (PTSD) presented in the fifth edition of the Diagnostic and Statistical Manual of Mental Disorder (DSM-5; American Psychiatric Association [APA], 2013), the 11th version of the International Classification of Diseases (ICD-11; World Health Organization [WHO], 2018) includes a refined description of PTSD and introduces the diagnosis of complex PTSD (CPTSD) to the diagnostic nomenclature for the first time. Complex PTSD is listed under the parent category "Disorders Specifically Associated with Stress" (Code 6B41), and diagnosis requires traumatic exposure and at least one symptom from each of six clusters. The first three clusters, which are shared with PTSD (Code 6B40), are "reexperiencing in the here and now," "avoidance of traumatic reminders," and "sense of current threat." The the sec-

Correspondence concerning this article should be addressed to Philip Hyland, Department of Psychology, Maynooth University, Kildare, Ireland. E-mail: philip.hyland@mu.ie

(c) 2019 International Society for Traumatic Stress Studies. View this article online at wileyonlinelibrary.com

DOI: $10.1002 /$ jts.22444 ond three clusters, reflecting disturbances in self-organization (DSO), are "affective dysregulation," "negative self-concept," and "difficulties in forming and maintaining interpersonal relationships." Complex PTSD also requires that the PTSD and DSO symptoms cause significant functional impairment. The introduction of an operational definition of CPTSD along with a valid and reliable measure of CPTSD in the form of the International Trauma Questionnaire (ITQ; Cloitre et al., 2018) has allowed researchers to test the construct validity of CPTSD. Considerable data have accrued in support of the factorial, concurrent, convergent, discriminant, incremental, and predictive validity of ICD-11 PTSD and CPTSD (see Brewin et al., 2017); however, a common critique of CPTSD is that it lacks discriminant validity from borderline personality disorder (BPD), due to conceptual overlap in symptom presentation (Resick et al., 2012).

Indeed, BPD and CPTSD do share conceptual overlap in the type of problems that are included in each diagnosis-namely, difficulties in affect regulation, self-concept, and interpersonal relationships. However, as noted by Cloitre, Garvert, Weiss, 
Carlson, and Bryant (2014), there are important phenomenological differences in how these symptoms manifest across the disorders. In BPD, self-concept difficulties reflect an unstable sense of self, whereas in CPTSD, they reflect a persistent negative sense of self. Relational difficulties in BPD are characterized by volatile patterns of interactions, whereas in CPTSD, they reflect a persistent tendency to avoid relationships. Affect dysregulation in BPD is characterized by fears of abandonment and self-harming or suicidal behavior, whereas in CPTSD, it reflects difficulties in maintaining emotional equilibrium. There are other relevant features that differentiate CPTSD from BPD; most notably, CPTSD requires trauma exposure for diagnosis as well as the presence of trauma-specific PTSD symptoms.

To date, three studies have tested the discriminant validity of ICD-11 CPTSD and BPD symptoms. Two of these studies, based on predominately female trauma-exposed samples, employed latent class analysis and identified four (Cloitre et al., 2014) and five (Frost, Hyland, Shevlin, \& Murphy, 2018) distinct classes of trauma survivors. Distinct ICD-11 PTSD and CPTSD classes emerged in both samples, along with a nonsymptomatic class. Moreover, Cloitre et al. (2014) identified a distinct BPD class, whereas Frost et al. (2018) identified comorbid PTSD/BPD and CPTSD/BPD classes. These studies showed that although DSO and BPD symptoms are conceptually similar, one set of symptoms can occur independently of the other. A similar result was reported by Knefel, Tran, and Lueger-Schuster (2016), who employed network analysis among a sample of adult survivors of childhood abuse $(N=219)$. Their results showed that PTSD and DSO symptoms formed a strongly connected network of symptoms, with BPD symptoms weakly connected to this network. Collectively, these studies indicate that CPTSD and BPD symptoms are related to one another but that they are also meaningfully distinguishable from each other.

This picture of CPTSD and BPD symptoms as both related and distinct from one another is inconsistent with a traditional categorical, or "diagnostic," model of psychopathology. In the traditional categorical model of psychopathology, psychiatric disorders and their constituent symptoms are described as entirely discrete constructs. However, dimensional models of psychopathology, such as the hierarchical taxonomy of psychopathology (HiTOP: Kotov et al., 2017), represent psychiatric disorders and their constituent symptoms as highly correlated constructs. These correlations exist because the disorders and their symptoms are manifestations of a small number of underlying latent dimensions of psychopathology. Specifically, HiTOP describes PTSD and DSO symptoms as observable indicators of "internalizing" psychopathology and BPD symptoms as indicators of internalizing and "antagonistic externalizing" psychopathology. Furthermore, the HiTOP model predicts that these purportedly discrete disorders (i.e., PTSD, CPTSD, and BPD) will share similar risk factors because of their common latent structure (Kotov et al., 2017). Consistent with the predictions of the HiTOP model, childhood interpersonal trauma has been identified as a common risk factor for both BPD and DSM-IV PTSD (Zanarini, Laudate, Frankenburg, Reich, \& Fitzmaurice, 2011).

The existing empirical (Cloitre et al., 2014; Frost et al., 2018; Knefel et al., 2016) and theoretical (Kotov et al., 2017) literature means that it is unreasonable to expect PTSD, DSO, and BPD symptoms to be entirely distinct from one another. This poses a methodological challenge when attempting to test the discriminant validity of $I C D-11$ CPTSD and BPD symptoms. Confirmatory factor analysis (CFA) is typically used to assess discriminant validity; however, in a traditional CFA model, variation in an observable symptom (e.g., flashbacks) is attributable to one latent variable (e.g., reexperiencing), with the remaining variation attributable to systematic or random measurement error. Traditional CFA models do not permit cross-factor loadings, and this restriction is especially pertinent when testing the discriminant validity of conceptually similar constructs, such as CPTSD and BPD. This is because symptom indicators are recognized to be fallible representations of their underlying latent variable and, therefore, in circumstances in which conceptually similar constructs are being modeled, it is highly probable that some degree of variation in a given indicator will be due to a nonspecified latent variable. The presence of unacknowledged cross-factor loadings, even of an extremely small magnitude (e.g., .10), has been shown to bias model fit results and inflate the true correlation between latent variables in a model (Asparouhov, Muthén, \& Morin, 2015; Marsh, Morin, Parker, \& Kaur, 2014). Asparouhov and Muthén (2009) developed a framework called "exploratory structural equation modeling" (ESEM) in order to overcome this limitation of CFA. In ESEM, key elements of CFA (model falsification), EFA (inclusion of cross-factor loadings), and SEM (inclusion of exogenous and/or endogenous variables) are combined within a single approach, thus permitting complex models to be accurately represented and empirically tested in an unbiased manner (Marsh et al., 2014). The ability to recognize the conceptually overlapping nature of CPTSD and BPD symptoms via the inclusion of cross-factor loadings while simultaneously recognizing their phenomenological distinctiveness and their independent associations with exogenous variables makes ESEM an ideal statistical framework with which to test the discriminant validity of these symptoms.

We hypothesized that an ESEM model with three latent variables would provide an optimal representation of the latent structure of CPTSD and BPD symptoms and that the three latent variables would reflect the symptom clusters of PTSD, DSO, and BPD. We also expected nontrivial cross-factor loadings for symptoms on each latent variable, reflecting the conceptual overlap between many of these symptoms. In line with the predictions of the HiTOP model, we hypothesized that (a) more frequent trauma exposure would be associated with higher scores on each latent variable, (b) interpersonal and noninterpersonal trauma would be positively associated with PTSD (see Ben-Ezra et al., 2018; Shevlin et al., 2018), and (c) childhood interpersonal trauma would be related to all latent variables but would be most strongly associated with DSO (Cloitre, 
Garvert, Brewin, Bryant, \& Maercker, 2013; Karatzias et al., 2017).

\section{Method}

\section{Participants and Procedure}

Participants were recruited from an online research panel that is representative of the entire United Kingdom (UK) adult population. Panel members were randomly recruited through probability-based sampling to ensure representativeness to the UK population. Several inclusion criteria were used to recruit participants for the current study, including that (a) participants were born in the United Kingdom; (b) were aged 18 years or older; and (c) screened positive for at least one traumatic life event, assessed using the Life Events Checklist-Revised (LEC-5; described in the Measures section). Ethical approval was granted by the research ethics committee of the institution to which the lead author was affiliated at the time of the survey. No inducements or incentives were offered for participation. In total, 2,653 panel members were assessed and 1,051 met the inclusion criteria, for a participation rate of $39.6 \%$. The sample participants in this study were a random half of these 1,051 participants $(N=546)$ who completed the measure of BPD symptoms using a Likert-scale response format (additional details are provided in the Measures section). The mean age of the sample was 47.21 years ( $S D=14.94$, range: $18-83$ years). The majority of participants were female $(n=377,69.0 \%)$, currently in a committed relationship ( $n=370,67.8 \%)$, completed university ( $n=339,62.1 \%$ ), and currently employed ( $n$ $=306,56.0 \%)$. Nearly half of the sample grew up in an urban area $(n=260,47.6 \%)$, and $18.3 \%(n=187)$ emigrated at some point in their lifetime. The random half of the sample selected for this study did not differ from the random half of the sample excluded from this study on any demographic factors.

\section{Measures}

Borderline personality disorder. Symptoms of BPD were measured using 14 items that were based on the BPD screening module of the Structured Clinical Interview for DSM-IV Axis II disorders (SCID-II; First, Gibbon, Spitzer, Williams, \& Benjamin, 1997). As BPD symptoms are not typically measured using self-report scales, we were interested in determining if these symptoms were better assessed using a binary response format $(0=n o, 1=y e s)$ or a 5 -point Likert-scale response format $(0=$ never, $1=$ rarely, $2=$ sometimes, $3=$ often, $4=$ always). In the binary format, participants were asked to indicate whether each of the 14 statements was true. In the Likert-scale response format, participants were asked to indicate how often each statement was true. When responses to the items were compared across the two response formats, the proportion of individuals who responded positively in the binary response condition was approximately equal to the proportion of individuals who responded with a rating of rarely or more (i.e., a score of 1 or above) on the Likert scale for each item. In other words, when individuals were asked to use the responses of yes or no to indicate if a particular BPD symptom was present, they appeared to respond affirmatively even if they rarely experienced such an event. We thus concluded that the responses to the binary items were not useable given that the endorsement of features of a personality disorder demands that they be experienced on a regular basis. Consequently, we focused the analyses for the current study on the Likert scale items only. As the primary objective of the current study was to model the latent structure of CPTSD and BPD symptoms, operating the analyses as the "trait" level was advantageous in preserving variation in responses. The internal reliability of the 14 BPD (Likert scale) items among the current sample was excellent, Cronbach's $\alpha=.90$.

Complex PTSD. Symptoms of CPTSD were measured using the ITQ (Cloitre et al., 2018), which is a self-report measure based on ICD-11 criteria for CPTSD and PTSD. The ITQ first screens for an individual's index traumatic event and the time elapsed since this event occurred. Six items measure PTSD symptoms, and six items measure DSO symptoms; in the current sample, the Cronbach's alpha values for PTSD and DSO items were .91 and .92 , respectively. Additionally, three items measure functional impairment in social, occupational, and other important areas of life that is associated with both sets of symptoms. Individuals respond to the PTSD items in terms of how much they have been bothered by that symptom over the past month and DSO items in terms of how they typically feel, think about themselves, and relate to others. The PTSD and DSO items are measured using a 5-point Likert scale ranging from 0 (not at all) to 4 (extremely), and the present analyses were based on these Likert-scale responses.

For the purposes of calculating diagnostic rates, PTSD and DSO symptoms were deemed to be present based on scores of 2 or higher (i.e., moderately or more). A diagnosis of PTSD requires the presence of at least one symptom from each PTSD cluster (reexperiencing, avoidance, and sense of threat) as well as endorsement of at least one indicator of functional impairment associated with these symptoms. A diagnosis of CPTSD requires the presence of at least one symptom from each of the six PTSD and DSO clusters (reexperiencing, avoidance, sense of threat, affective dysregulation, negative self-concept, and disturbances in relationships) as well as endorsement of at least one indicator of functional impairment associated with the PTSD and DSO symptoms, respectively. The validity and reliability of the ITQ have been demonstrated within the full sample (Cloitre et al., 2018), half of whom were included in the present study. The prevalence rates of PTSD and CPTSD in the full sample were $5.3 \%$ and $12.9 \%$, respectively, and the prevalence rates among the random half of the sample used in this study were $5.1 \%$ and $13.0 \%$, respectively.

Trauma exposure. Exposure to traumatic events was measured using a revised version of the LEC-5 (Weathers et al., 
2013). In the present study, we assessed childhood (i.e., before 18 years of age) and adulthood (i.e., at or after 18 years of age) exposure to the 16 different traumatic events included on the LEC-5. Participants indicated on a yes (1) or no (0) basis if they had directly experienced each traumatic event during both developmental periods. The mean number of lifetime traumatic events experienced was $3.77(S D=4.09)$, and $37.0 \%$ of respondents reported having experienced four or more traumatic events in their lifetime. Following Ehring and Quack's (2010) scoring guidelines, we developed summed scores for noninterpersonal (i.e., natural disaster, fire or explosion, transportation accident, serious accident at work or home or during recreational activity, exposure to toxic substance, life-threatening illness or injury, severe human suffering, sudden and violent death, sudden and unexpected death of someone close to you), and interpersonal (i.e., physical assault, assault with a weapon, sexual assault, other unwanted or uncomfortable sexual experiences, combat or exposure to a war zone, captivity, serious injury and/or harm and/or death you caused to someone else) trauma in childhood and in adulthood. Scores for noninterpersonal trauma in childhood and in adulthood ranged from 0 to 9 , and scores for interpersonal trauma in childhood and in adulthood ranged from 0 to 7 .

\section{Data Analysis}

Descriptive statistics for interpersonal and noninterpersonal trauma exposure as well as PTSD, DSO, and BPD symptoms were calculated first. Sex differences in PTSD, DSO, and BPD symptoms were assessed by means of independent samples $t$ tests, and bivariate associations between age and PTSD, DSO, and BPD symptoms were assessed using Pearson correlation tests. These analyses were conducted using SPSS (Version 25).

The ESEM analyses were conducted in Mplus (Version 7.4; Muthén \& Muthén, 2013), and the models were estimated using the mean and variance-adjusted weighted least squares (WLSMV) estimator, which is appropriate for ordered categorical indicators (Flora \& Curran, 2004). The geomin rotation method was used, and the extracted factors were allowed to correlate. Six ESEM models were tested, with one to six latent factors extracted, in order to determine the optimal number of latent variables required to explain the covariation between the 26 PTSD, DSO, and BPD symptoms. As is standard in an ESEM framework, the models were estimated with, in this case, six exogenous covariates: sex $(0=$ male, $1=$ female $)$; age; and cumulative scores for childhood noninterpersonal trauma, childhood interpersonal trauma, adulthood noninterpersonal trauma, and adulthood interpersonal trauma. All covariates were treated as observed variables.

The adequacy of each model was assessed in relation to a number of goodness-of-fit indices (Hu \& Bentler, 1999): A nonsignificant chi-square result indicates excellent model fit; however, this test is positively related to sample size and, therefore, a significant result should not lead to the rejection of a model (Tanaka, 1987). Comparative fit index (CFI) and
Tucker-Lewis index (TLI) values greater than .90 indicate adequate fit, and CFI and TLI values greater than .95 indicate excellent fit. Additionally, root mean square error of approximation (RMSEA) values less than .08 and less than .06 indicate adequate and excellent model fit, respectively. Like exploratory factor analysis models, ESEM models will always produce improved model fit as more latent variables are extracted. Therefore, it is recommended that models are compared using an indicator of fit that includes a correction for model parsimony (see Morin, Arens, \& Marsh, 2016). Following standard guidelines (Marsh et al., 2009, 2014), we relied on changes in the RMSEA (i.e., $\triangle$ RMSEA) to determine the optimal number of latent variables that should be extracted. A change in RMSEA of greater than .015 indicates significant improvement in model fit compared to a model with one less factor (Chen, Curran, Bollen, Kirby, \& Paxton, 2008). There were no missing data present in the present analyses.

\section{Results}

\section{Descriptive Statistics}

Table 1 presents the descriptive statistics for interpersonal and noninterpersonal trauma in childhood and adulthood as well as the total symptom scores for the PTSD, DSO, and BPD clusters. Female participants had significantly higher symptom levels for PTSD, $t(544)=4.27, p<.001, d=0.40$; DSO, $t(544)$ $=2.22, p=.027, d=0.21$; and BPD $t(544)=2.95, p=.003$, $d=0.27$, compared to male participants. Age was significantly, negatively, and moderately correlated with symptoms of PTSD, $r=-.31, p<.001$; DSO, $r=-.40, p<.001$; and BPD, $r=$ $-.42, p<.001$.

\section{Exploratory Structural Equation Modeling Results}

The ESEM model fit results are presented in Table 2. The oneand two-factor models were rejected as they yielded unsatisfactory model fit results. The three-factor model had acceptable fit according to the CFI, TLI, and RMSEA indices, and this model was statistically superior to the two-factor model, $\triangle \mathrm{RMSEA}=$ .017 . The extraction of a fourth factor was not supported as the change in RMSEA of .013 was below the critical threshold for the acceptance of improved model fit. We therefore concluded that an ESEM model with three latent variables was the optimal representation of the latent structure of the 26 PTSD, DSO, and BPD symptoms. The factor loadings and factor correlations derived from this model are presented in Table 3.

The six PTSD items loaded significantly, $p<.001$, and strongly on Factor $1, M \lambda=.70$. Additionally, four items from the DSO cluster and one item from the BPD cluster cross-loaded significantly and weakly on this factor, $\lambda \mathrm{s}<.20$. This factor was therefore labeled PTSD. The six DSO items loaded positively and significantly, $p<.001$, on Factor 2 . All of the DSO items loaded on this factor robustly with the exception of item AD1 (i.e., emotional reactivity), $M \lambda=.74$. Four PTSD items and 
Table 1

Descriptive Statistics for All Continuous Variables

\begin{tabular}{lcccrr}
\hline Variable & $M$ & $95 \%$ CI & $M d n$ & $S D$ & Range \\
\hline Posttraumatic stress disorder symptoms & 6.59 & {$[6.03,7.15]$} & 4.00 & 6.64 & $0-24$ \\
Disturbance in self-organization symptoms & 8.13 & {$[7.55,8.70]$} & 6.50 & 6.84 & $0-24$ \\
Borderline personality disorder symptoms & 15.80 & {$[14.75,16.86]$} & 13.00 & 12.29 & $0-56$ \\
Childhood noninterpersonal trauma & 0.81 & {$[0.70,0.91]$} & 0 & 1.28 & $0-9$ \\
Childhood interpersonal trauma & 0.81 & {$[0.70,0.91]$} & 0 & 1.23 & $0-7$ \\
Adulthood noninterpersonal trauma & 1.39 & {$[1.26,1.51]$} & 1.00 & 1.49 & $0-9$ \\
Adulthood interpersonal trauma & 0.77 & {$[0.67,0.88]$} & 0 & 1.24 & $0-7$ \\
\hline
\end{tabular}

nine BPD items cross-loaded significantly on this factor, and the majority of these loadings were weak, with the exception except of item BPD11 (i.e., feeling empty inside), which had a moderately sized factor loading. This factor was therefore labeled DSO. Finally, the 14 BPD items loaded significantly, $p<$ .001 , and moderately-to-strongly on Factor $3, M \lambda=.71$. The two items related to sense of threat from the PTSD cluster and the two items related to affective dysregulation from the DSO cluster cross-loaded significantly, positively, and robustly onto this factor. This factor was therefore labeled BPD. The three factors were significantly, $p s<.001$, and positively correlated with one another. The PTSD factor correlated weakly with the DSO and BPD factors, $r \mathrm{~s}=.24$ and .23 , respectively; and the $\mathrm{BPD}$ and DSO factors were strongly correlated, $r=.63$.

The standardized regression coefficients between each covariate and the PTSD, DSO, and BPD latent variables are presented in Table 4. Childhood interpersonal trauma was positively associated with PTSD, DSO, and BPD, and the effects were of a similar magnitude. Adulthood noninterpersonal trauma was positively associated with DSO and BPD, and the effect sizes were similar. Adulthood interpersonal trauma was positively associated with BPD. Younger age was associated with higher scores on each latent variable, and the effect was strongest for BPD, followed by DSO and then PTSD. Gender was significantly associated with PTSD, with female participants scoring higher than male participants.

\section{Discussion}

The current study examined the phenomenological similarities and differences between $I C D-11$ CPTSD symptoms and BPD symptoms. Based on the existing evidence regarding the nature of the association between these symptoms (Cloitre et al., 2014; Frost et al., 2018; Knefel et al., 2016) and guided by empirically supported dimensional models of psychopathology (Andrews et al., 2009; Goodkind et al., 2015; Kotov et al., 2017; Taylor et al., 2018), we hypothesized that CPTSD and BPD symptoms would be related to one another while also possessing satisfactory discriminant validity. Consistent with our primary study hypothesis, the ESEM results demonstrated that the latent structure of these symptoms was best explained by three factors that predominantly, but not exclusively, captured the shared variance between the symptom clusters of PTSD, DSO, and BPD.

The pattern of factor loadings across the three latent variables revealed much about the symptom level similarities and differences between CPTSD and BPD. Overall, the symptoms unique to the DSO factor included emotional avoidance and interpersonal withdrawal, whereas those for the BPD factor included emotional and interpersonal reactivity. The DSO affective dysregulation symptoms-emotional reactivity and emotional numbing-loaded strongly on the BPD factor, although numbing was stronger for DSO, whereas reactivity was stronger

Table 2

Exploratory Structural Equation Modeling Model Fit Results for Models With One to Six Latent Factors

\begin{tabular}{lccccccc}
\hline Model & $\chi^{2}$ & $d f$ & CFI & TLI & RMSEA & 90\% CI RMSEA & k-1 Factor $\Delta$ RMSEA \\
\hline One factor & $4,158^{* * *}$ & 449 & .835 & .823 & .123 & {$[.120, .126]$} & - \\
Two factors & $2,454^{* * *}$ & 418 & .909 & .896 & .094 & {$[.091, .098]$} & .029 \\
Three factors & $\mathbf{1 , 6 5 0}^{* * *}$ & $\mathbf{3 8 8}$ & $\mathbf{. 9 4 4}$ & $\mathbf{. 9 3 0}$ & $\mathbf{. 0 7 7}$ & {$[.073, .081]$} & $.060, .068]$ \\
Four factors & $1,162^{* * *}$ & 359 & .964 & .952 & .064 & .017 & .013 \\
Five factors & $8,62^{* * *}$ & 331 & .976 & .966 & .054 & $.059]$ & .010 \\
Six factors & $6,60^{* * *}$ & 304 & .984 & .975 & .046 & {$[.042, .051]$} & .008 \\
\hline
\end{tabular}

Note. $N=546 . d f=$ degrees of freedom; CFI = comparative fit Index; TLI = Tucker-Lewis index; RMSEA = root mean square error of approximation. ${ }^{a}$ Change in RMSEA value for each model relative to the model with one fewer factor; best-fitting model is formatted in bold.

**** $p<.001$. 
Table 3

Factor Loadings and Factor Correlations for the Three-Factor Model Derived From the Exploratory Structural Equation Modeling Analysis

\begin{tabular}{|c|c|c|c|c|c|c|}
\hline \multirow[b]{2}{*}{ Variable } & \multicolumn{2}{|c|}{ Factor 1: PTSD } & \multicolumn{2}{|c|}{ Factor 2: DSO } & \multicolumn{2}{|c|}{ Factor 3: BPD } \\
\hline & $\lambda$ & $S E$ & $\lambda$ & $S E$ & $\Lambda$ & $S E$ \\
\hline \multicolumn{7}{|l|}{ PTSD symptoms } \\
\hline RE1. Upsetting dreams & $.64^{*}$ & .04 & $.24^{*}$ & .06 & .08 & .06 \\
\hline RE2. Flashback & $.70^{*}$ & .03 & $.23^{*}$ & .07 & .02 & .05 \\
\hline AV1. Avoidance of internal reminders & $.75^{*}$ & .04 & $.35^{*}$ & .07 & -.05 & .05 \\
\hline AV2. Avoidance of external reminders & $.73^{*}$ & .03 & $.31^{*}$ & .06 & -.01 & .02 \\
\hline TH1. Being on guard & $.70^{*}$ & .05 & -.07 & .04 & $.42^{*}$ & .06 \\
\hline TH2. Jumpy/Startled & $.67^{*}$ & .05 & .00 & .01 & $.44^{*}$ & .06 \\
\hline \multicolumn{7}{|l|}{ DSO symptoms } \\
\hline AD1. Emotional reactivity & $.17^{*}$ & .05 & $.20^{*}$ & .05 & $.46^{*}$ & .05 \\
\hline AD2. Emotional numbing & $.12^{*}$ & .04 & $.54^{*}$ & .04 & $.29^{*}$ & .05 \\
\hline NSC1. Feel like a failure & $-.11^{*}$ & .03 & $.98^{*}$ & .02 & -.01 & .02 \\
\hline NSC2. Feel worthless & $-.10^{*}$ & .03 & $.99^{*}$ & .03 & .02 & .02 \\
\hline DR1. Feel cut off from others & .00 & .02 & $.85^{*}$ & .04 & .05 & .05 \\
\hline DR2. Difficulty staying close to others & .00 & .03 & $.85^{*}$ & .04 & .01 & .05 \\
\hline \multicolumn{7}{|l|}{ BPD symptoms } \\
\hline BPD1. Frantic someone close will leave & $.16^{*}$ & .07 & .04 & .06 & $.66^{*}$ & .04 \\
\hline BPD2. Relationship ups and downs & .04 & .07 & .09 & .07 & $.69^{*}$ & .05 \\
\hline BPD3. Sudden change of self-image & .09 & .07 & $.16^{*}$ & .06 & $.72^{*}$ & .04 \\
\hline BPD4. Sense of self dramatically changes & .07 & .07 & $.19^{*}$ & .06 & $.73^{*}$ & .04 \\
\hline BPD5. Don't know who you really are & .07 & .06 & $.22^{*}$ & .06 & $.64^{*}$ & .04 \\
\hline BPD6. Sudden changes in your life plans & .01 & .04 & $.17^{*}$ & .06 & $.61^{*}$ & .05 \\
\hline BPD7. Impulsive behaviors & .01 & .04 & $.14^{*}$ & .06 & $.61^{*}$ & .05 \\
\hline BPD8. Tried to hurt or kill yourself & -.11 & .10 & -.01 & .05 & $.89^{*}$ & .03 \\
\hline BPD9. Self-harm behavior & -.06 & .10 & -.07 & .08 & $.86^{*}$ & .05 \\
\hline BPD10. Sudden mood changes & -.05 & .06 & $.25^{*}$ & .06 & $.69^{*}$ & .04 \\
\hline BPD11. Feel empty inside & -.04 & .04 & $.52^{*}$ & .04 & $.45^{*}$ & .04 \\
\hline BPD12. So angry you lose control & -.04 & .09 & -.01 & .07 & $.85^{*}$ & .04 \\
\hline BPD13. Violent when angry & -.06 & .12 & $-.29^{*}$ & .10 & $.93^{*}$ & .06 \\
\hline BPD14. Paranoia/dissociation & .10 & .07 & $.19^{*}$ & .06 & $.65^{*}$ & .05 \\
\hline \multicolumn{7}{|l|}{ Factor correlations } \\
\hline Factor 1 (PTSD) & 1 & & & & & \\
\hline Factor 2 (DSO) & $.24^{*}$ & .07 & 1 & & & \\
\hline Factor 3 (BPD) & $.23^{*}$ & .11 & $.63^{*}$ & .04 & 1 & \\
\hline
\end{tabular}

Note. $N=546$. PTSD $=$ posttraumatic stress disorder; $\mathrm{DSO}=$ disturbances in self-organization; $\mathrm{BPD}=$ borderline personality disorder; $\lambda=$ standardized factor loading.

${ }^{*} p<.05$.

for BPD. In the DSO factor, the relationship difficulty-related symptoms of feeling cut off from others and having difficulty staying close to others did not load on the BPD factor; conversely, fears of abandonment and relationship ups-and-downs loaded on the BPD factor but not the DSO factor. Symptoms that were unique to the BPD factor were suicidal and self-injurious behaviors as well as anger to the point of losing control. These findings replicate results reported by Cloitre et al. (2014) and give some confidence in what may be emerging as the "phe- nomenological signatures" that distinguish CPTSD and BPD. Such discoveries can advance clinical knowledge. For example, BPD symptoms including sudden changes in self-image and in mood loaded on the DSO factor, but weakly, suggesting that this type of reactivity may be present in individuals with CPTSD but that it is not likely to be a salient feature of the disorder. The BPD factor included the two DSO items related to emotional reactivity and numbing, but other items, such as suicidal and self-harming behaviors, loaded very strongly only on the BPD 
Table 4

Standardized Regression Coefficients for the Three-Factor Model Derived From the Exploratory Structural Equation Modeling Analysis

\begin{tabular}{|c|c|c|c|c|c|c|}
\hline \multirow[b]{2}{*}{ Variable } & \multicolumn{2}{|c|}{$\begin{array}{c}\text { Factor } 1 \\
\text { PTSD }\end{array}$} & \multicolumn{2}{|c|}{$\begin{array}{c}\text { Factor } 2 \\
\text { DSO }\end{array}$} & \multicolumn{2}{|c|}{$\begin{array}{c}\text { Factor } 3 \\
\text { BPD }\end{array}$} \\
\hline & $\beta$ & $S E$ & $\beta$ & $S E$ & $\beta$ & $S E$ \\
\hline Sex (female) & $.19^{* * *}$ & .05 & .01 & .04 & .05 & .04 \\
\hline Age & $-.11^{*}$ & .05 & $-.32^{* * *}$ & .04 & $-.36^{* * *}$ & .04 \\
\hline Child noninterpersonal trauma & .01 & .05 & .00 & .05 & .00 & .05 \\
\hline Child interpersonal trauma & $.24^{* * *}$ & .05 & $.23^{* * *}$ & .05 & $.27^{* * *}$ & .05 \\
\hline Adult noninterpersonal trauma & .07 & .06 & $.09^{*}$ & .05 & $.11^{*}$ & .05 \\
\hline Adult interpersonal trauma & .07 & .06 & .04 & .05 & $.13^{* *}$ & .05 \\
\hline
\end{tabular}

Note. $N=546$. PTSD = posttraumatic stress disorder; DSO = disturbances in self-organization; BPD = borderline personality disorder.

${ }^{*} p<.05{ }^{* *} p<.01{ }^{* * *} p<.001$.

factor, suggesting these latter items are central to BPD but not CPTSD.

The associations between the PTSD, DSO, and BPD latent variables and the six covariates indicated additional areas of similarity and difference between the constructs. Consistent with an extensive literature (e.g., Jonas et al., 2011), we found that exposure to a larger number of different childhood interpersonal traumas was similarly strongly associated with the PTSD, DSO, and BPD latent variables. This finding was inconsistent with our hypothesis that childhood interpersonal traumas would be most strongly associated with the DSO latent variable. Increased DSO scores were also associated with elevated levels of adulthood noninterpersonal trauma, whereas increased BPD scores were associated with elevated levels of both adulthood noninterpersonal and adulthood interpersonal traumatic exposure. Both ICD-11 CPTSD and BPD have been shown to be more impairing and severe forms of psychopathology than ICD-11 PTSD (Cloitre et al., 2014). Given that these data are cross-sectional, it is unknown whether trauma exposure in adulthood is a cause or consequence of these disorders. Research findings have consistently shown that PTSD symptoms and disturbances in self-regulation create risk for additional traumatic exposure (e.g., Iverson et al., 2013; Messman-Moore, Brown, \& Koelsch, 2005). It may be that DSO and BPD symptoms reflect the more extreme end of an underlying continuum of disturbance and may additionally create more risk in adulthood for additional trauma exposure. Our multivariate results suggest that having experienced childhood interpersonal trauma is the dominant risk factor for all indicators of psychopathology and that the occurrence of trauma during adulthood is associated with more severe indicators of psychopathology (i.e., DSO and BPD symptoms).

These data have implications for considering different types of trauma as potential differential risk factors for the diagnoses of PTSD, CPTSD and BPD. Several studies have found that cumulative childhood trauma exposure is a better predictor of CPTSD than PTSD (e.g., Cloitre et al., 2013; Cloitre et al., current issue; Karatzias et al., 2016; Palic et al., 2016). The current data are not necessarily in contradiction with these findings. It may very well be that when the naturally occurring latent variables are forced into categorical designations representing the diagnostic constructs of CPTSD (i.e., PTSD plus DSO symptoms) versus PTSD (i.e., PTSD symptoms alone), the former will include a larger number of individuals who have experienced childhood trauma. This provides useful information from a diagnostic perspective and potentially from a treatment perspective. On the other hand, the strong association between trauma exposure and the BPD latent variable might suggest that BPD as a diagnosis is not most strongly differentiated from CPTSD or PTSD by trauma history. Nevertheless, CPTSD and PTSD not only require a traumatic experience but also a specific set of trauma-related consequences, namely the symptoms of reexperiencing, avoidance, and sense of threat, which strongly distinguish these disorders from BPD. The BPD latent variable is associated with trauma exposure but not symptoms of reexperiencing, avoidance, and sense of threat. This suggests that there are many pathways by which childhood trauma can influence adult symptom expression and that there may be some (as yet to be identified) developmental trajectories that distinguish the development of BPD from CPTSD and PTSD.

In addition to the associations with trauma exposure, younger age was associated with higher levels of PTSD, DSO, and BPD; this is a result consistent with an extensive literature demonstrating that younger individuals are more likely to experience multiple forms of psychopathology, including PTSD (Frueh, Grubaugh, Acierno, Elhai, Cain, \& Magruder, 2007) and BPD (Arens et al., 2013). We also found that women had higher levels of PTSD than men, which is consistent with what has been reported in much of the trauma literature (Tolin \& Foa, 2006).

There were several limitations to the present study that should be considered. First, this study was based on a trauma-exposed sample of the general population; therefore, the generalizability of these results to the entire population is unclear. However, given that $70.4 \%$ of the world's population has experienced at least one traumatic life event (Benjet et al., 2016), the current findings are meaningful. Second, future studies should 
seek to replicate these findings using clinician-administered assessments of BPD and CPTSD symptoms. Given that there is no gold standard self-report measure of BPD symptoms available and that data from the excluded half of the current sample revealed a tendency for respondents to positively endorse symptoms of BPD even when they were rarely experienced, clinician-administered measures of BPD symptoms would strengthen the confidence in these results. Nonetheless, given the time and resource constraints associated with using clinician-administered measures in large, epidemiological surveys, data obtained from self-reports offer useful information. Third, the generalizability of these results to non-Englishspeaking countries is unknown. As international applicability is a core organizing principle of the $I C D-11$, cross-cultural replication will be important.

In conclusion, the current results obtained from a traumaexposed sample of the general population in the United Kingdom add to data from the United States (Cloitre et al., 2014; Frost et al., 2018) and Austria (Knefel et al., 2016) that support the discriminant validity of $I C D-11$ CPTSD and BPD symptoms. The picture that is emerging of the relationship between CPTSD and BPD appears to be similar to that which is evident between any other two psychiatric disorders: The constituent symptoms share some conceptual similarity, there is a moderate-to-strong correlation between the constructs at a dimensional level, the constructs share similar associations with external risk variables, and there is a high level of diagnostic comorbidity (see Hyland Shevlin, Fyvie, \& Karatzias, 2018). The expectation that CPTSD and BPD should be entirely distinct from one another at a symptomatic, dimensional, or diagnostic level is entirely untenable when considered in light of empirically supported dimensional models of psychopathology (e.g., HiTOP: Kotov et al., 2017). We believe that clinicians would therefore benefit most from research that identifies the specific symptoms and risk factors that most effectively differentiate these constructs from one another. The current study has highlighted endogenous (e.g., affective dysregulation symptoms) and exogenous (e.g., childhood interpersonal trauma exposure and younger age) variables that are common across the latent variables of PTSD, DSO, and BPD, as well as endogenous (e.g., sense of threat, negative self-concepts, disturbed interpersonal relationships, suicidality, and anger/volatility) and exogenous (e.g., sex, interpersonal trauma in adulthood) variables that are unique to each. Future research should continue to clarify the unique phenomenological signatures of each of these disorders.

\section{References}

American Psychiatric Association. (2013). Diagnostic and statistical manual of mental disorders (5th ed.). Washington, DC: Author.

Andrews, G., Goldberg, D. P., Krueger, R. F., Carpenter, W. T., Hyman, S. E., Sachdev, P., \& Pine, D. S. (2009). Exploring the feasibility of a meta-structure for $D S M-V$ and $I C D-11$ : Could it improve utility and validity?: Paper 1 of 7 of the thematic section: A proposal for a metastructure for DSM-V and ICD-11. Psychological Medicine, 39, 1993-2000. https://doi.org/10.1017/s0033291709990250
Asparouhov, T., \& Muthén, B. (2009). Exploratory structural equation modeling. Structural Equation Modeling, 16, 397-438. https://doi.org/ $10.1080 / 10705510903008204$

Asparouhov, T., Muthén, B., \& Morin, A. J. S. (2015). Bayesian structural equation modeling with cross-loadings and residual covariances: Comments on Stromeyer et al. Journal of Management, 41, 1561-1577. https://doi.org/10.1177/0149206315591075

Arens, E. A., Stopsack, M., Spitzer, C., Appel, K., Dudeck, M., Völzke, H., ... Barnow, S. (2013). Borderline personality disorder in four different age groups: A cross-sectional study of community residents in Germany. Journal of Personality Disorders, 27, 196-207. https://doi.org/ 10.1521/pedi.2013.27.2.196.

Ben-Ezra, M., Karatzias, T., Hyland, P., Brewin, C. R., Cloitre, M., Bisson, J. I., ... Shevlin, M. (2018). Posttraumatic stress disorder (PTSD) and complex PTSD (CPTSD) as per ICD-11 proposals: A population study in Israel. Depression and Anxiety, 35, 264-274. https://doi. org/10.1002/da.22723

Benjet, C., Bromet, E., Karam, E. G., Kessler, R. C., McLaughlin, K. A., Ruscio, A. M., ... Koenen, K. C. (2016). The epidemiology of traumatic event exposure worldwide: Results from the World Mental Health Survey Consortium. Psychological Medicine, 46, 327-343. https://doi.org/10.1017/S0033291715001981

Brewin, C. R., Cloitre, M., Hyland, P., Shevlin, M., Maercker, A., Bryant, R. A., ... Reed, G. M. (2017). A review of current evidence regarding the ICD-11 proposals for diagnosing PTSD and complex PTSD. Clinical Psychology Review, 58, 1-15. https://doi.org/10.1016/j.cpr.2017.09.001

Chen, F., Curran, P. J., Bollen, K. A., Kirby, J., \& Paxton, P. (2008). An empirical evaluation of the use of fixed cutoff points in RMSEA test statistic in structural equation models. Sociological Methods \& Research, 36, 462494. https://doi.org/10.1177/0049124108314720

Cloitre, M., Garvert, D. W., Brewin, C. R., Bryant, R. A., \& Maercker, A. (2013). Evidence for proposed ICD-11 PTSD and complex PTSD: A latent profile analysis. European Journal of Psychotraumatology, 4. http://doi.org/10.3402/ejpt.v4i0.20706

Cloitre, M., Garvert, D. W., Weiss, B., Carlson, E. B., \& Bryant, R. A. (2014). Distinguishing PTSD, complex PTSD, and borderline personality disorder: A latent class analysis. European Journal of Psychotraumatology, 5. http://doi.org/10.3402/ejpt.v5.25097

Cloitre, M., Shevlin, M., Brewin, C. R., Bisson, J. I., Roberts, N. P., Maercker, A., ... Hyland, P. (2018). The International Trauma Questionnaire (ITQ): Development of a self-report measure of ICD-11 PTSD and Complex PTSD. Acta Psychiatrica Scandinavica, 138, 536-546. https://doi.org/10.1111/acps.12956

Ehring, T., \& Quack, D. (2010). Emotion regulation difficulties in trauma survivors: The role of trauma type and PTSD symptom severity. Behavior Therapy, 41, 587-598. https://doi.org/10.1016/j.beth.2010.04.004

First, M. B., Gibbon, M., Spitzer, R. L., Williams, J. B. W., \& Benjamin, L. S. (1997). Structured Clinical Interview for DSM-IV Axis II Personality Disorders Self-Report. Washington, DC: American Psychiatric Association.

Flora, D. B., \& Curran, P. J. (2004). An empirical evaluation of alternative methods of estimation for confirmatory factor analysis with ordinal data. Psychological Methods, 9, 466-491.https://doi.org/10.1037/1082-989X.9.4. 466

Frost, R., Hyland, P., Shevlin, M., \& Murphy, J. (2018). Distinguishing Complex PTSD from Borderline Personality Disorder among individuals with a history of sexual trauma; a latent class analysis. European Journal of Trauma \& Dissociation. https://doi.org/10.1016/j.ejtd.2018.08.004

Frueh, B. C., Grubaugh, A. L., Acierno, R., Elhai, J. D., Cain, G., \& Magruder, K. M. (2007). Age differences in posttraumatic stress disorder, psychiatric 
disorders, and healthcare service use among veterans in veterans affairs primary care clinics. American Journal of Geriatric Psychiatry, 15, 660 672. https://doi.org/10.1097/01.JGP.0000260855.42209.31

Goodkind, M., Eickhoff, S. B., Oathes, D. J., Jiang, Y., Chang, A., JonesHagata, L. B., ... Etkin, A. (2015). Identification of a common neurobiological substrate for mental illness. JAMA Psychiatry, 72, 305-315. https://doi.org/10.1001/jamapsychiatry.2014.2206

Hu, L., \& Bentler, P. M. (1999). Cutoff criteria for fit indexes in covariance structure analysis: Conventional criteria versus new alternatives. Structural Equation Modeling, 6, 1-55. https://doi.org/10.1080/10705519909540118

Hyland, P., Shevlin, M., Fyvie, C., \& Karatzias, T. (2018). Posttraumatic stress disorder (PTSD) and complex PTSD (CPTSD) in DSM-5 and ICD-11: Clinical and behavioral correlates. Journal of Traumatic Stress, 31, 174-180. https://doi.org/10.1002/jts.22272

Iverson, K. M., Litwack, S. D., Pineles, S. L., Suvak, M. K., Vaughn, R. A., \& Resick, P. A. (2013). Predictors of intimate partner violence revictimization: The relative impact of distinct PTSD symptoms, dissociation, and coping strategies. Journal of Traumatic Stress, 26, 102-110. https://doi.org/10.1002/jts.21781

Jonas, S., Bebbington, P., McManus, S., Meltzer, H., Jenkins, R., Kuipers, E., ... Brugha, T. (2011). Sexual abuse and psychiatric disorder in England: results from the 2007 Adult Psychiatric Morbidity Survey. Psychological medicine, 41, 709-19. https://doi.org/10.1017/S003329171000111X

Karatzias, T., Shevlin, M., Fyvie, C., Hyland, P., Efthimiadou, E., Wilson, D., ... Cloitre, M.(2017). Evidence of distinct profiles of posttraumatic stress disorder (PTSD) and complex posttraumatic stress disorder (CPTSD) based on the new ICD-11 Trauma Questionnaire (ICD-TQ). Journal of Affective Disorders, 207, 181-187. https://doi.org/10.1016/j.jad.2016.09.032

Knefel, M., Tran, U. S., \& Lueger-Schuster, B. (2016). The association of posttraumatic stress disorder, complex posttraumatic stress disorder, and borderline personality disorder from a network analytical perspective. Journal of Anxiety Disorders, 43, 70-78. https://doi.org/10.1016/j.janxdis.2016.09.002

Kotov, R., Krueger, R. F., Watson, D., Achenbach, T. M., Althoff, R. R., Bagby, R. M., ... Zimmerman, M. (2017). The Hierarchical Taxonomy of Psychopathology (HiTOP): A dimensional alternative to traditional nosologies. Joural of Abnormal Psychology, 126, 454-477. https://doi.org/10.1037/abn0000258

Marsh, H. W., Morin, A. J. S., Parker, P., \& Kaur, G. (2014). Exploratory structural equation modeling: an integration of the best features of exploratory and confirmatory factor analysis. Annual Review of Clinical Psychology, 10, 85-110. https://doi.org/10.1146/annurev-clinpsy-032813-153700

Marsh, H. W., Muthén, B., Asparouhov, T., Lüdtke, O., Robitzsch, A., Morin, A. J. S., \& Trautwein, U. (2009). Exploratory structural equation modeling, integrating CFA and EFA: Application to students' evaluations of university teaching. Structural Equation Modeling: A Multidisciplinary Journal, 16, 439-476. https://doi.org/10.1080/10705510903008220
Messman-Moore, T. L., Brown, A. L., \& Koelsch, L. E. (2005). Posttraumatic symptoms and self-dysfunction as consequences and predictors of sexual revictimization. Journal of Traumatic Stress, 18, 253-261. https://doi.org/10.1002/jts.20023

Morin, A. J. S., Arens, A., \& Marsh, H. (2016). A bifactor exploratory structural equation modeling framework for the identification of distinct sources of construct-relevant psychometric multidimensionality. Structural Equation Modeling, 23, 116-39. https://doi.org/10.1080/10705511.2014. 961800

Muthén, L. K., \& Muthén, B. O. (2013). Mplus user's guide (7th ed.). Los Angeles, CA: Muthén \& Muthén.

Palic, S., Zerach, G., Shevlin, M., Zeligman, Z., Elklit, A., \& Solomon, Z. (2016). Evidence of complex posttraumatic stress disorder (CPTSD) across populations with prolonged trauma of varying interpersonal intensity and ages of exposure. Psychiatry Research, 246, 692-699. http://doi.org/10.1016/j.psychres.2016.10.062

Resick, P. A., Bovin, M. J., Calloway, A. L., Dick, A. M., King, M. W., Mitchell, K. S., ... Wolf, E. J. (2012). A critical evaluation of the complex PTSD literature: Implications for DSM-5. Journal of Traumatic Stress, 25, 239-249. https://doi.org/10.1002/jts.21699

Shevlin, M., Hyland, P., Vallières, F., Bisson, J., Makhashvili, N., Javakhishvili, ... Roberts, B. (2018). A comparison of DSM-5 and ICD11 Posttraumatic Stress Disorder rates and comorbidity: An analysis of the Ukrainian Internally Displaced Person's Mental Health Survey. Acta Psychiatrica Scandinavica, 137, 138-147. https://doi.org/10.1111/acps. 12840

Tanaka, J. S. (1987). "How big is big enough?”: Sample size and goodness of fit in structural equation models with latent variables. Child Development, 58, 134-146. https://doi.org/10.1111/j.1467-8624.1987.tb03495.x

Taylor, M. J., Martin, J., Lu, Y., Lundström, S., Larsson, H., \& Lichtenstein, P. (2018). Association of genetic risk factors for psychiatric disorders and traits of these disorders in a Swedish population twin sample. JAMA Psychiatry. https://doi.org/10.1001/jamapsychiatry.2018.3652

Tolin, D. F., \& Foa, E. B. (2006). Sex differences in trauma and posttraumatic stress disorder: A quantitative review of 25 years of research. Psychological Bulletin, 132, 959-92. https://doi.org/10.1037/0033-2909.132.6.959

Weathers, F. W., Litz, B. T., Keane, T. M., Palmieri, P. A., Marx, B. P., \& Schnurr, P. P. (2013). The PTSD Checklist for DSM-5 (PCL-5). Scale available from the National Center for PTSD at www.ptsd.va.gov

World Health Organization. (2018). The $I C D-11$ for mortality and morbidity statistics. Retrieved from https://icd.who.int/browse11/l-m/en

Zanarini, M. C., Laudate, C. S., Frankenburg, F. R., Reich, D. B., \& Fitzmaurice, G. (2011). Predictors of self-mutilation in patients with borderline personality disorder: A 10-year follow-up study. Journal of Psychiatric Research, 45, 823-828. https://doi.org/10.1016/j.jpsychires.2010. 10.015 